\title{
短嘴金丝燕回声定位叫声特征
}

\author{
王 斌 ${ }^{1,4}$, 马建章 ${ }^{1}$, 陈 毅 $^{2}$, 谭梁静 ${ }^{2}$, 刘 奇 $^{2}$, 沈琪琦 ${ }^{2}$, 廖庆义 ${ }^{3}$, 张礼标 ${ }^{2, *}$ \\ 1. 东北林业大学 野生动物资源学院, 黑龙江 哈尔滨 $150040 ; 2$. 广东省昆虫研究所, 广东 广州 $510260 ; 3$. 壸瓶山国家级自然保护区, \\ 湖南 石门 $415300 ; 4$. 湖南师范大学 生命科学学院, 湖南 长沙 410008
}

\begin{abstract}
摘要: 2012 年 6 月, 对湖南省石门县壸瓶山国家级自然保护区神景洞短嘴金丝燕的回声定位叫声进行研究, 在黑暗山 洞内使用录音仪器录制其自由飞行状态的声音后使用声音软件进行分析。短嘴金丝燕捕食归巢时，快速飞入洞口，在洞 内有光区域不发声, 到达洞内黑暗区域后开始发出回声定位叫声, 且飞行速度减慢。声音分析结果表明其回声定位叫声 为双脉冲组的噪声脉冲串型(noise burst), 组内脉冲间隔很短 $[(6.6 \pm 0.42) \mathrm{ms}]$, 组间脉冲间隔较长 $[(99.3 \pm 3.86) \mathrm{ms}]$, 两者 差异显著 $(P<0.01)$ 。对比第一、第二脉冲声音参数发现, 主频和脉冲时程差异不显著, 第一、第二脉冲主频分别为

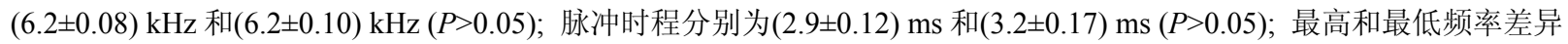
显著, 第一、第二脉冲最高频率分别为 $(20.1 \pm 1.10) \mathrm{kHz}$ 和 $(15.4 \pm 0.98) \mathrm{kHz}(P<0.01)$, 最低频率分别为 $(3.7 \pm 0.12) \mathrm{kHz}$ 和 $(4.0 \pm 0.09) \mathrm{kHz}(P<0.05)$; 第一脉冲频宽 $((16.5 \pm 1.17) \mathrm{kHz})$ 宽于第二脉冲 $((11.4 \pm 1.01) \mathrm{kHz})(P<0.01)$; 且第一脉冲能量 $[(-32.5 \pm 0.60) \mathrm{dB}]$ 高于第二脉冲 $[(-35.2 \pm 0.94) \mathrm{dB}](P<0.05)$ 。另外, 短嘴金丝燕在黑暗山洞内的回声定位叫声还包含了部 分超声波，最高频率可达 $33.2 \mathrm{kHz}$ 。
\end{abstract}

关键词: 短嘴金丝燕; 回声定位叫声; 超声波; 脉冲组

中图分类号: Q959.7+35; Q958 文献标志码: A 文章编号：0254-5853-(2013)01-0008-06

\section{Echolocation calls of free-flying Himalayan swiftlets (Aerodramus brevirostris)}

\author{
Bin WANG ${ }^{1,4}$, Jian-Zhang MA ${ }^{1}$,Yi CHEN ${ }^{2}$, Liang-Jing TAN ${ }^{2}$, Qi LIU², Qi-Qi SHEN ${ }^{2}$, \\ Qing-Yi LIAO ${ }^{3}$, Li-Biao ZHANG ${ }^{2, *}$ \\ 1. College of Wildlife Resources, Northeast Forestry University, Harbin 150040, China; 2. Guangdong Entomological Institute, Guangzhou 510260, \\ China; 3. Hupingshan National Nature Reserve, Shimen 415300, China; 4. School of Life Science, Hunan Normal University, Changsha 41000, China
}

\begin{abstract}
Here, we present our findings of free-flying echolocation calls of Himalayan swiftlets (Aerodramus brevirostris), which were recorded in Shenjing Cave, Hupingshan National Reserve, Shimen County, Hunan Province in June 2012, using Avisoft-UltraSoundGate 116(e). We noted that after foraging at dusk, the Himalayan swiftlets flew fast into the cave without clicks, and then slowed down in dark area in the cave, but with sounds. The echolocation sounds of Himalayan swiftlets are broadband, double noise burst clicks, separated by a short pause. The inter-pulse intervals between double clicks $(99.3 \pm 3.86$ $\mathrm{ms})$ were longer than those within double clicks $(6.6 \pm 0.42 \mathrm{~ms})(P<0.01)$. With the exception of peak frequency, between $6.2 \pm 0.08 \mathrm{kHz}$ and $6.2 \pm 0.10 \mathrm{kHz},(P>0.05)$ and pulse duration $2.9 \pm 0.12 \mathrm{~ms}, 3.2 \pm 0.17 \mathrm{~ms},(P>0.05)$ between the first and second, other factors - maximum frequency, minimum frequency, frequency bandwidth, and power-were significantly different between the clicks. The maximum frequency of the first pulse $(20.1 \pm 1.10 \mathrm{kHz})$ was higher than that of second $(15.4 \pm 0.98 \mathrm{kHz})$ $(P<0.01)$, while the minimum frequency of the first pulse $(3.7 \pm 0.12 \mathrm{kHz})$ was lower than that of second $(4.0 \pm 0.09 \mathrm{kHz})$ $(P<0.05)$; resulting in the frequency bandwidth of the first pulse $(16.5 \pm 1.17 \mathrm{kHz})$ longer than that of second $(11.4 \pm 1.01 \mathrm{kHz})$ $(P<0.01)$. The power of the first pulse $(-32.5 \pm 0.60 \mathrm{~dB})$ was higher than that of second $(-35.2 \pm 0.94 \mathrm{~dB})(P<0.05)$. More importantly, we found that Himalayan swiftlets emitted echolocation pulses including ultrasonic sound, with a maximum frequency reaching $33.2 \mathrm{kHz}$.
\end{abstract}

Key words: Aerodramus brevirostris; Echolocation; Ultrasonic sound; Double clicks

\footnotetext{
收稿日期: 2012-09-03; 接受日期: 2012-10-31

基金项目: 广东省昆虫研究所优秀青年人才基金 (GDEI-yxqn201101); 广州市珠江科技新星专项 (2011J2200027)

*通信作者 (Corresponding author), E-mail: zhanglb@gdei.gd.cn

第一作者简介: 王斌, 博士研究生, 讲师, 主要从事鸟类生态学研究工作, E-mail: southtigerhn@163.com
} 
鸟类中，仅新热带的油鸱 (Steatornithidae: Steatornis caripensis) 和部分古热带的金丝燕种类 (Apodidae)进化出回声定位导航能力。它们均栖息 在洞穴内, 在弱光或无光环境中飞行时发出可听的 滴答回声定位叫声(clicks)(Griffin, 1958; Medway, 1959)。研究表明, 金丝燕使用回声定位叫声主要是 为了在黑暗山洞内的飞行过程中躲避障碍物, 而不 是像蝙蝠那样除了使用回声定位进行导航之外, 还 用于捕食猎物等(Collins \& Murphy, 1994; Cranbrook \& Medway, 1965; Fenton, 1975; Griffin \& Suthers, 1970; Griffin \& Thompson, 1982; Medway, 1962, 1967)。

大部分使用回声定位叫声的金丝燕种类发出 的滴答声为宽频双脉冲组, 组内脉冲间隔较短, 而 组间脉冲间隔较长(Griffin \& Suthers, 1970; Medway \& Pye, 1977; Suthers \& Hector, 1982)。通常, 第二脉 冲能量 $>$ 第一脉冲, 两个脉冲的主频主要为 $2 \sim 8$ $\mathrm{kHz}$ ，但是，同一物种不同个体之间，甚至同一个体 不同状态下的脉冲特征都可能存在明显变化 (Suthers \& Hector, 1982)。迄今为止，仅发现大金丝 燕(Aerodramus maximus)(Medway 1959; Medway \& Pye 1977)和阿蒂乌金丝燕(A. sawtelli)(Fullard et al, 1993)的回声定位叫声为单独脉冲而非双脉冲组。

金丝燕外形特征较为相似, 物种鉴别难度较大 (Chantler \& Driessens, 1995; Chantler et al, 2000), 且其巢穴特征所提供的有关系统进化关系的信息 也相对很少(Lee et al, 1996)。而由于回声定位叫声 的有无提供了一些相对重要的系统进化信息, 因此 之前被用于区别金丝燕属(Aerodramus) 与侏金丝燕 属(Collocalia) 和瀑布雨燕属(Hydrochous)(Brooke, 1972; Medway \& Pye, 1977)。侏金丝燕属包括 3 个 物种, 其中白腹金丝燕 $(C$. esculenta $)$ 和穴金丝燕 $(C$. linchi) 已经被证实缺乏回声定位能力(Cranbrook \& Medway, 1965; Fenton, 1975; Medway, 1967), 而侏 金丝燕 (C. troglodytes) 是否具备回声定位能力之前 一直备受争议(Chantler \& Driessens, 1995; Chantler et al, 2000), 直至 2004 年其所具备的回声定位能力 才被 Price et al 所证实。因此, 目前看来, 将回声定 位能力作为金丝燕属的分类特征是有一定限制的。 通过分子数据, Price et al (2004)还证实, 金丝燕(金 丝燕属和侏金丝燕属)为单起源, 即目前的金丝燕 属和侏金丝燕属有着共同的祖先。那么, 金丝燕属 所有物种和侏金丝燕属的侏金丝燕均具备回声定
位能力的解释可能有两种推测, 即: 回声定位能力 的进化早于人们之前的想像，而在之后的侏金丝燕 属大部分物种中被丢失; 或者, 回声定位能力在这 两个金丝燕属中被分别进化出来(Price et al, 2004)。

MacKinnon et al (2000)记载中国分布的金丝燕 有短嘴金丝燕 (A. brevirostris) 和戈氏金丝燕 $(C$. germani)(作者也提出可将该种归入金丝燕属 Aerodramus)。而 Zheng (2011)则指出，中国分布有 3 种金丝燕，即: 爪哇金丝燕 (A.fuciphagus)、短嘴金 丝燕 (A. brevirostris)和大金丝燕 (A. maximus)。本 文沿用后者的分类系统，认为中国分布的金丝燕均 为金丝燕属(Aerodramus)。关于这三种金丝燕的回 声定位叫声国内、外均有一些报道 (Li et al, 2007; Price et al, 2004; Thomassen \& Povel, 2006)。本文使 用 Avisoft 声音录制仪器对湖南省石门县壶瓶山国 家级自然保护区神景洞的短嘴金丝燕进行声音录 制，分析其在黑暗环境中自由飞行状态下的声音特 征, 并与之前的文献报道进行了对比。Avisoft 声音 录制仪器可以较好的录制可听声、超声波和次声波, 为更准确了解短嘴金丝燕的回声定位叫声提供了 较为理想的仪器设备。

\section{1 材料与方法}

研究地点位于湖南省石门县壶瓶山国家级自 然保护区神景洞 $\left(\mathrm{N} 30^{\circ} 3.3^{\prime}\right.$, E110³5.1'，海拔 1650 $\mathrm{m})$, 该洞穴为石灰岩结构，同时至少还栖息着 2 种 蝙蝠，中华菊头蝠 (Rhinolophus sinicus) 和中菊头 蝠(R.affinis)。短嘴金丝燕在洞穴内的栖息巢穴在不 同深度 $(80 \sim 620 \mathrm{~m})$ 均有发现，但主要集中在 80 、 430 和 $620 \mathrm{~m}$ 。 2012 年 6 月, 使用 AvisoftUltraSoundGate 116(e)录音仪器(德国)对短嘴金丝 燕在山洞内自由飞行时进行实时录制, 采样频率为 $250 \mathrm{kHz}$ ，声音数据直接保存在笔记本电脑中。由于 神景洞内的短嘴金丝燕种群数量不多 ( 30 只), 且 巢穴分布在洞内的不同位置，因此，大部分时候可 以录制到单个个体在洞穴内自由飞行的声音; 同时 选择不同的录制位置以保证能够录制到不同个体 的声音。在距离洞口 80、430 和 $620 \mathrm{~m}$ (均为完全黑 暗)的三个位置录音，每个位置持续录音 $1 \mathrm{~h}$, 录音 设备距离录音对象 2 3 m。使用 Avisoft- SASLab Pro 软件(Version 5.2, Avisoft Bioacoustics, 德国)进 行声音特征分析。参数设置为: 快速傅里叶变换 (FFT length) $=256$ points, 哈明窗口 (Hamming 
window), 频率分辨率(frequency resolution) $=977 \mathrm{~Hz}$, 时间分辨率(time resolution) $=0.512 \mathrm{~ms}$ 。在不同地段 的声音文件中各随机抽取 10 个声音文件(共 30 个), 从每个声音文件中抽取连续的 30 个叫声进行分析, 其平均值代表一个样本的叫声。由于无法确定所分 析声音文件的个体, 因此, 本研究所说的样本量仅 指声音文件的样本量, 不代表个体样本量。分析的 主要特征参数为: 主频率、最低频率、最高频率、 脉冲时程和脉冲间隔 (从前一个脉冲的开始到下一 个脉冲的开始)。脉冲时程和脉冲间隔通过示波图测 量, 主频率通过能谱图测量, 最低和最高频率通过 声谱图测量, 频宽 (bandwidth, BW) 通过最低频率 与最高频率计算。使用 SPSS 11.0 对数据进行双尾 $t$-test, 统计分析的显著性水平为 $P<0.05$, 结果以 mean $\pm S E$ 表示。

\section{2 结 果}

通过行为观察发现，短嘴金丝燕进入洞口时的 飞行速度很快, 且不发出叫声; 进入弱光带后飞行 速度逐渐减慢, 并且在减慢的过程中开始发出叫声;
到达洞内黑暗区域后，飞行速度明显减慢。

使用软件分析短嘴金丝燕在洞内黑暗环境中 3 个不同地段发出的声音(每个地段 $n=10$ )。结果表明, 短嘴金丝燕在黑暗山洞内使用回声定位进行导航, 发出的声波大部分为可听声; 不同地段的回声定位 叫声参数无差异(one-way ANOVA, $P>0.05$ ), 因此, 在后续分析中将 3 个地段的声音进行合并。从图 1 可见, 短嘴金丝燕的回声定位叫声为双脉冲组(即 两个脉冲组成一个脉冲组)的噪声脉冲串型(noise burst), 组内脉冲间隔很短 $[(6.6 \pm 0.4) \mathrm{ms}]$, 组间脉冲 间隔较长 $[(99.3 \pm 3.9) \mathrm{ms}$, 两者差异显著 $(t=23.89$, $P<0.01$ )(表 1)。对比第一脉冲(组内的第一个脉冲) 与第二脉冲 (组内第二个脉冲)的声音参数发现, 主 频和脉冲时程差异不显著, 第一和第二脉冲主频分 别为(6.2 \pm 0.1$)$ 和(6.2 \pm 0.1$) \mathrm{kHz}(t=0.57, P>0.05)$, 脉冲 时程分别为 $(2.9 \pm 0.1)$ 和 $(3.2 \pm 0.2) \mathrm{ms}(t=-1.55$, $P>0.05)$; 两者的最高和最低频率差异显著, 第一、 第二脉冲的最高频率分别为 $(20.1 \pm 1.1) \mathrm{kHz}$ 和 $(15.4 \pm 1.0) \mathrm{kHz}(t=3.24, P<0.01)$, 而最低频率分则别 为 $(3.7 \pm 0.1) \mathrm{kHz}$ 和 $(4.0 \pm 0.1) \mathrm{kHz}(t=-2.32, P<0.05)$,

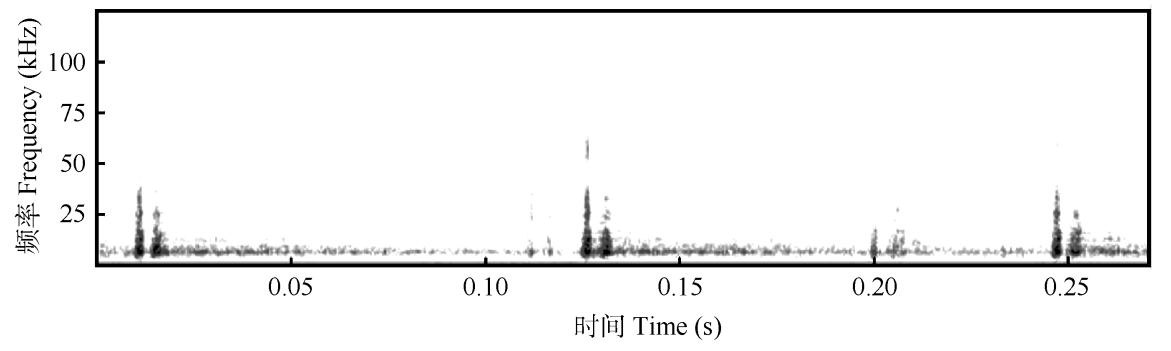

图 1 短嘴金丝燕在黑暗洞穴内自由飞行的回声定位叫声语谱图

Figure 1 Spectrograms of echo-clicks of Himalayan swiftlets (Aerodramus brevirostris) free-flying in the dark cave

表 1 短嘴金丝燕在黑暗洞穴内自由飞行的回声定位叫声第一和第二脉冲参数比较

Table 1 Comparison echo-clicks' first and second pulses of Himalayan swiftlets (Aerodramus brevirostris) free-flying in the dark cave

\begin{tabular}{|c|c|c|c|}
\hline 参数 Parameter & 第一脉冲 First pulse & 第二脉冲 Second pulse & $t$-test \\
\hline 主频 PF(kHz) & $6.2 \pm 0.1(5.5 \sim 8.4)$ & $6.2 \pm 0.1(5.4 \sim 8.6)$ & $0.57^{\mathrm{ns}}$ \\
\hline 能量 Power(-dB) & $32.5 \pm 0.6(26.6 \sim 38.9)$ & $35.2 \pm 0.9(23.8 \sim 47.4)$ & $-2.46^{*}$ \\
\hline 最高频率 FMAX(kHz) & $20.1 \pm 1.1(12.4 \sim 33.2)$ & $15.4 \pm 1.0(9.0 \sim 28.9)$ & $3.24 * *$ \\
\hline 最低频率 FMIN(kHz) & $3.7 \pm 0.1(2.3 \sim 4.6)$ & $4.0 \pm 0.1(3.2 \sim 5.0)$ & $-2.32 *$ \\
\hline 频宽 BW(kHz) & $16.5 \pm 1.2(7.8 \sim 29.5)$ & $11.4 \pm 1.0(5.4 \sim 24.6)$ & $3.30 * *$ \\
\hline 脉冲时程 PD(ms) & $2.9 \pm 0.1(1.7 \sim 4.8)$ & $3.2 \pm 0.2(1.8 \sim 5.6)$ & $-1.55^{\mathrm{ns}}$ \\
\hline 脉冲间隔 IPI(ms) & $6.6 \pm 0.4(2.5 \sim 11.5)$ & $99.3 \pm 3.9(67.4 \sim 153.0)$ & $-23.89 * *$ \\
\hline
\end{tabular}

第一脉冲的脉冲间隔即组内脉冲间隔, 第二脉冲的脉冲间隔即组间脉冲间隔 $(n=30)$ 。

The IPI of first pulse is the IPI within double clicks, IPI of second pulse is the IPI between double clicks $(n=30)$. PF: peak frequency, FMAX: maximum frequency, FMIN: minimum frequency, BW: frequency bandwidth, PD: pulse duration, IPI: interpulse interval.

${ }^{\text {ns: }} P>0.05, *: P<0.05, * *: P<0.01$ 。 
从而导致第一脉冲的频宽 $[(16.5 \pm 1.2) \mathrm{kHz}]>$ 第二脉 冲 $[(11.4 \pm 1.0) \mathrm{kHz}](t=3.30, P<0.01)$ 。此外, 第一脉冲

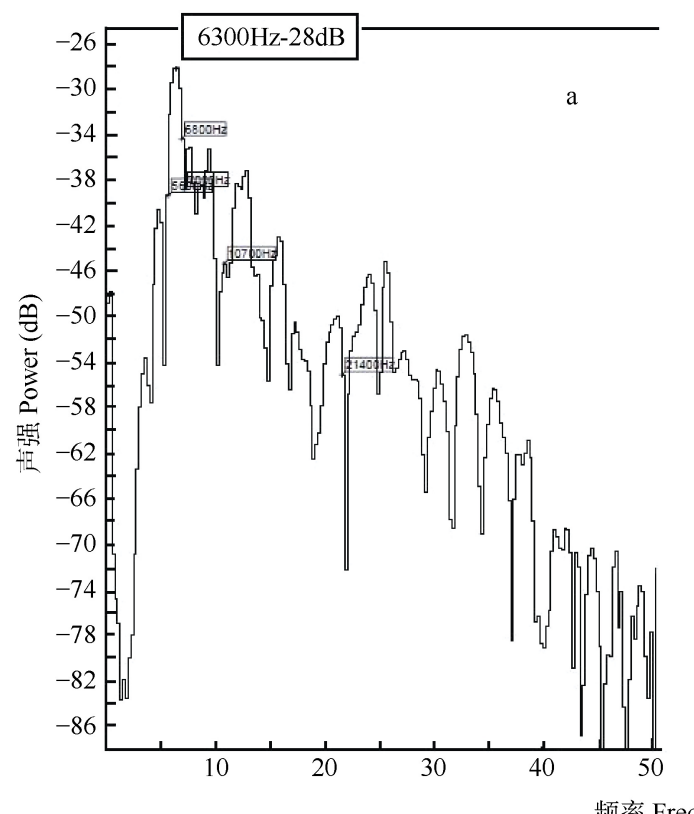

图 2 同一脉冲组内第一脉冲(a)和第二脉冲(b)的能谱图

Figure 2 Frequency with energy distribution graphs (LPC graphs) of first (a) and second (b) clicks from the same double clicks group of Himalayan swiftlets (Aerodramus brevirostris)

通过对声波的分析还发现，短嘴金丝燕在黑暗 山洞内为进行导航定位而发出的超声波虽然大部 分能量集中在可听声范围(主频 $6.2 \mathrm{kHz}$ ), 但仍包 含了部分超声波 (即频率 $>20 \mathrm{kHz}$ ), 如, 第一脉冲 最高频率平均为 $(20.1 \pm 1.1) \mathrm{kHz}$ (最高可达 $33.2 \mathrm{kHz}$ ), 第二脉冲最高频率平均为 $(15.4 \pm 1.0) \mathrm{kHz}$ (最高可达 $28.9 \mathrm{kHz})($ 表 1)。

\section{3 讨 论}

野外观察发现, 湖南石门县壸瓶山国家级自然 保护区的短嘴金丝燕栖息在石灰岩洞穴内，与两种 蝙蝠共栖，栖息巢穴在不同深度均有发现 $(80 \sim 620$ $\mathrm{m})$ 。短嘴金丝燕傍晚捕食归巢时，通常成群在洞口 附近上空盘旋; 然后逐个快速飞入洞穴内(Medway, 1962; Thomassen \& Povel, 2006), 在光线较好的洞 口范围内不发出声音, 进入弱光带后逐渐发出声音, 飞行速度减慢, 到达洞内黑暗区域后使用连续的叫 声进行导航，且飞行速度明显减慢。

通过分析短嘴金丝燕在黑暗洞穴内自由飞行 的声音, 表明该物种的回声定位叫声为双脉冲组的 噪声脉冲串型。Thomassen \& Povel (2006)对多种金
的能量 $[(-32.5 \pm 0.6) \mathrm{dB}]$ 亦 $>$ 第二脉冲 $[(-35.2 \pm 0.9)$ $\mathrm{dB}](t=-2.46, P<0.05)$ (图 2)。



丝燕进行录音, 其中也包括了短嘴金丝燕, 分析结 果发现，金丝燕属物种发出的回声定位叫声基本均 为双脉冲组型。通过对比发现，除了组内脉冲间隔， 本研究结果中的其他声音参数均高于 Thomassen \& Povel (2006)的结果, 特别是最高频率。从最高频率 范围来看(表 1), 我们录制的短嘴金丝燕回声定位 叫声的最高频率包含了超声波部分 $(>20 \mathrm{kHz}$ ), 而 Thomassen \& Povel (2006)报道的短嘴金丝燕最高 频率仅为 $5 \sim 6 \mathrm{kHz}$ 。同时, 较高的最高频率也导致 我们录制声音的频宽较宽(第一、二脉冲分别为 16.5 $\mathrm{kHz}$ 和 $11.4 \mathrm{kHz}$, 而 Thomassen \& Povel (2006)的分 别为 $2.7 \mathrm{kHz}$ 和 $3.8 \mathrm{kHz}$ )。这种明显的差异可能是由 于录音环境和设备的不同所致(Price \& Lanyon, 2002; Price et al, 2004)。Thomassen \& Povel (2006) 使用的是普通录音机, 可能只适合录制可听声, 而 本研究使用的是较为专业的录音设备 [AvisoftUltraSoundGate 116(e)], 所录制声音的频率范围更 大; 此外, 本研究录制的是短嘴金丝燕在黑暗洞穴 内自由飞行的回声定位叫声, 而 Thomassen \& Povel (2006)在文中未介绍其录音环境。Thomassen \& Povel (2006) 同时还报道了多种金丝燕属物种, 


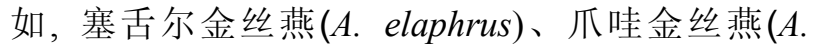
fuciphagus)、大金丝燕(A. maximus)、苔巢金丝燕 $(A$. salanganus)、澳大利亚金丝燕 (A.terraereginae)、纯 色金丝燕 (A. vanikorensis) 和火山金丝燕 $(A$. vulcanorum) 的回声定位叫声特征, 其主频均较低 (2.3 $8.5 \mathrm{kHz})$, 最高频率也未超过 $16 \mathrm{kHz}$ 。其统计 分析发现, 不同物种间的声音差异显著, 并认为声 音的种间差异可能有利于种内进行社会声音交流。

白腰金丝燕(A.spodiopygius) 回声定位叫声的 第一脉冲弱于第二脉冲(Suthers \& Hector, 1982), 同 时, 在大部分金丝燕中, 第一脉冲的脉冲时程较短 (Thomassen \& Povel, 2006)。分子系统进化的证据也 证实, 单独脉冲的回声定位叫声可能是由双脉冲组 进化而来(Price et al, 2004)。本研究发现, 短嘴金丝 燕回声定位叫声与白腰金丝燕不同, 第一脉冲要强 于第二脉冲, 但是, 与大部分金丝燕物种类似, 第 一脉冲时程略短于第二脉冲 (但差异不显著)。

Suthers \& Hector (1982)录制的白腰金丝燕回声定位 叫声中大部分叫声的第一脉冲较弱, 脉冲时程也较 短; 两个脉冲的能量和时程也可能出现一致; 偶见 只出现一个脉冲(第一脉冲缺失); 而极少见第一脉 冲强于第二脉冲。本研究所录制的短嘴金丝燕回声 定位叫声，80\%(24/30)为第一脉冲较强, $20 \%(6 / 30)$ 为第二脉冲较强, 偶见仅出现一个脉冲 (缺失第二 个脉冲)。金丝燕通过鸣管发出回声定位叫声, Suthers \& Hector (1982)指出, 鸣管的生理结构以及 第一脉冲发出后的持续压力可能是导致第二脉冲 强于第一脉冲且时程较长的原因; 而本研究却发现, 短嘴金丝燕的回声定位叫声的声强方面恰好相反, 即第一脉冲较强。Suthers \& Hector (1982)还指出, 鸣管过早关闭, 导致没有足够的呼出气流用于产生 双脉冲中的第一脉冲, 从而使得白腰金丝燕第一脉 冲可能缺失, 仅出现单脉冲。而本研究中回声定位 叫声语谱图的分析结果却显示短嘴金丝燕缺失的 是第二脉冲, 对于该现象的解释, 需要进一步的研 究, 特别是对其发声机制的深入探讨。

Li et al (2007)采用数字录音机(Sony MD Z710; 频率范围 20 20 000 Hz) 和 Sony 公司的外接麦克风 在四川对短嘴金丝燕放飞单个个体进行录音, 分析 结果表明, 其声音频率在 $2.5 \sim 9.5 \mathrm{kHz}$, “单次叫 声 $~ 0.038 \mathrm{hms}$, 两次鸣叫峰值间隔 $\sim 0.15 \mathrm{~s}$ ”, 该结 果与本研究结果差异亦较大。我们认为, 录音设备
和环境可能是导致差异的主要原因。此外, 录音时 仪器与短嘴金丝燕的距离也可能限制了我们获取 更多声音特别是超声波部分的信息。声音频率越高, 在空气中的衰减越快(Fenton, 2001), 如果录音仪器 距离声源太远, 高频部分可能因在空气中的快速衰 减而丢失。此外, 从图 1 中的语谱图也可以看出, 声 波能量主要集中在 $\sim 6.2 \mathrm{kHz}$, 频率越高, 能量越弱, 这也可能是导致本研究中声音录制过程可能出现 高频部分更多丢失的原因。之前的研究一直都未录 制到此部分超声波(Li et al, 2007; Price et al, 2004; Thomassen \& Povel, 2006), 录音设备和距离是可能 的两个原因。因此, 虽然短嘴金丝燕的回声定位能 力可能不如具有回声定位能力的蝙蝠精准, 但是, 其回声定位叫声仍包含了部分超声波, 说明短嘴金 丝燕的回声定位能力可能优于预想。20世纪 80 年 代, 已有学者试图测量金丝燕回声定位叫声的灵敏 度。Smyth \& Roberts (1983)通过不同的行为实验指 出, 白腰金丝燕在黑暗环境中能探测到的金属丝直 径为 $10 \sim 20 \mathrm{~mm}$; 而 Griffin \& Thompson (1982)则 指出, 白腰金丝燕可以探测到并回避直径为 $6.3 \mathrm{~mm}$ 的金属丝。由此可见, 金丝燕的回声定位能力较好, 其回声定位叫声包含有超声波部分是有可能的。

金丝燕为日行性鸟类，具有大的眼睛，使用视 觉定位其昆虫食物(Medway，1962)。短嘴金丝燕的 回声定位叫声主频为 $6.2 \mathrm{kHz}$, 相对来说其声波的 波长较长, 可能不适用于捕捉小型昆虫(Griffin \& Suthers, 1970; Griffin \& Thompson, 1982; Langham, 1980; Medway, 1962, 1967, 1969), 因此, 短嘴金丝 燕的回声定位叫声可能仅适用于在黑暗环境中导 航。此外, 金丝燕的回声定位叫声虽为双脉冲组, 但由于脉冲组内的时间间隔很短, 人类听觉无法辨 别为两个脉冲, 因此我们听到的仍为单个脉冲。国 内对于金丝燕的研究相对较少, 而有些金丝燕物种 (如爪哇金丝燕)由于其经济价值高而被人类干扰， 导致其种群数量大幅度下降。因此, 对金丝燕生态 学方面的研究, 将有助于对该种群的进一步了解, 并且有助于加强其种群保护工作。

致谢: 感谢两位审稿专家的宝贵意见; 感谢湖 南石门县壼瓶山国家级自然保护区管理处给予的 野外调查帮助。 


\section{参考文献:}

Brooke RK. 1972. Generic limits in Old World Apodidae and Hirundinidae. Bull Br Ornith Club, 92: 53-57.

Chantler P, Driessens G. 1995. Swifts: A Guide to the Swifts and Treeswifts of the World. Sussex, UK: Pica Press.

Chantler P, Wells DR, Schuchmann KL. 2000. Family Apodidae (Swifts)// del Hoyo J, Elliot A, Sargatal J. Handbook of the Birds of the World. Vol. 5. Barcelona, Spain: Lynx Edicions, 338-457.

Collins CT, Murphy R. 1994. Echolocation acuity of the Palawan swiftlet (Aerodramus palawanensis). Avocetta, 17: 157-162.

Cranbrook E, Medway L. 1965. Lack of ultrasonic frequencies in the calls of swiftlets (Collocalia spp.). Ibis, 107(2): 258.

Fenton MB. 1975. Acuity of echolocation in Collocalia hirundinacea (Aves: Apodidae), with comments on the distributions of echolocating swiftlets and molossid bats. Biotropica, 7(1): 1-7.

Fenton MB. 2001. Bats (Revised Edition). New York: Facts on File, Inc.

Fullard JH, Barclay RMR, Thomas DW. 1993. Echolocation in free-flying Atiu swiftlets (Aerodramus sawtelli). Biotropica, 25(3): 334-339.

Griffin DR. 1958. Listening in the Dark. New Haven: Yale University Press.

Griffin DR, Suthers RA. 1970. Sensitivity of echolocation in cave swiftlets. Biological Bulletin, 139(3): 495-501.

Griffin DR, Thompson D. 1982. Echolocation by cave swiftlets. Behavioral Ecology and Sociobiology, 10(2): 119-123.

Langham N. 1980. Breeding biology of the Edible-nest Swiftlet Aerodramus fuciphagus. Ibis, 122(4): 447-461.

Lee PLM, Clayton DH, Griffiths R, Page RDM. 1996. Does behavior reflect phylogeny in swiftlets (Aves: Apodidae)? A test using cytochrome b mitochondrial DNA sequences. Proceedings of the National Academy of Sciences USA, 93(14): 7091-7096.

Li P, Chen FJ, Huang SJ, Zong H. 2007. Himalayan swiftlet's echolocation.
Sichuan Journal of Zoology, 26(1): 38-40. [李鹏，陈发军，黄时杰，宗浩. 2007. 短嘴金丝燕的回声定位机制与其归巢行为的探究. 四川动物, 26(1): 38-40.]

MacKinnon J, Phillipps K, He FQ. 2000. A Field Guide to the Birds of China (Chinese translation). Changsha: Hunan Education Press, 94. [约 翰·马敬能，卡伦·菲利普斯，何芬奇. 2000. 中国鸟类野外手册 (中文翻 译版). 长沙: 湖南教育出版社, 94.]

Medway L. 1962. The swiftlets (Collocalia) of Niah cave, Sarawak. Par 2. Ecology and the regulation of breeding. Ibis, 104(2): 228-245.

Medway L. 1967. The function of echonavigation among swiftlets. Animal Behavior, 15(4): 416-420.

Medway L. 1969. Studies on the biology of the Edible-nest swiftlets of South-East Asia. Mala Nat J, 22: 57-63

Medway L, Pye JD. 1977. Echolocation and the systematics of swiftlets, Chap. 19 // Stonehouse B, Perrins CM. Evolutionary Ecology. Baltimore: University Park Press, 225-238.

Price JJ, Lanon SM. 2002. Reconstructing the evolution of complex bird song in the oropendolas. Evolution, 56(7): 1514-1529.

Price JJ, Johnson KP, Clayton DH. 2004. The evolution of echolocation in swiftlets. Journal of Avian Biology, 35(2): 135-143.

Suthers RA, Hector DH. 1982. Mechanism for the production of echolocating clicks by the grey swiftlet, Collocalia spodiopygia. J Journal of Comparative Physiology, 148(4): 457-470.

Smyth DM, Roberts JR. 1983. The sensitivity of echolocation by the grey swiftlet Aerodramus spodiopygius. Ibis, 125(3): 339-345.

Thomassen HA, Povel GDE. 2006. Comparative and phylogenetic analysis of the echo clicks and social vocalizations of swiftlets (Aves: Apodidae). Biological Journal of Linnean Society, 88(4): 631-643.

Zheng GM. 2011. A Checklist of the Classification and Distribution of the Birds of China. $2^{\text {nd }}$ ed. Beijing: Science Press, 133-134. [郑光美. 2011. 中 国鸟类分类与分布名录. 2 版. 北京: 科学出版社, 133-134.] 